\title{
Grain-Orientation Related Surface Effects on Polycrystalline Tungsten Caused by Mechanical Polishing and Etching
}

\author{
Inge Uytdenhouwen ${ }^{1}$, Willy Vandermeulen ${ }^{1 *}$, Yevhen Zayachuk ${ }^{2}$ and Raymond Kemps ${ }^{3}$ \\ 1. Belgian Nuclear Research Centre, SCKCEN, Boeretang 200, 2400 Mol, Belgium \\ 2. Department of Materials, University of Oxford, Parks Road, Oxford OX1 3PH, UK and Culham Centre for Fusion Energy, \\ Culham Science Centre, Abingdon OX14 3DB, UK
}

3. Flemish Institute for Technological Research, Vito, Boeretang 200, 2400 Mol, Belgium

\begin{abstract}
The problem of obtaining a smoothly polished planar surface on polycrystalline tungsten is important for its applications as substrate in electronics as well as for preparing surfaces for research related to fusion reactor applications. In the latter case the classical metallographic polishing methods, using diamond abrasives, are generally used to prepare surfaces for the study of the interaction of the fusion plasma with tungsten. During a short study of the mechanical polishing of pure tungsten, it was observed that the material removal was different from grain to grain, giving rise to a non-planar surface. By polishing with $3 \mu \mathrm{m}$ diamond grit as an intermediate step a dot pattern develops which outlines the grain structure. The dots were shown to consist of small cracks. On further polishing with $1 \mu \mathrm{m}$ diamond the dot pattern disappears. However, it re-appears when polishing again with $3 \mu \mathrm{m}$ grit. Apparently these effects are caused by the orientation dependent mechanical interaction between the $3 \mu \mathrm{m}$ diamond particles and the tungsten crystal lattice. The coincidence of the surface dot patterns and the underlying grain structure could be clearly demonstrated by etching. Etching also demonstrated the presence of plastic deformation to a depth of the order of $4 \mu \mathrm{m}$. It is advised to further examine the sub-surface deformation layer in view of its effect on deuterium and tritium storage.
\end{abstract}

Key words: Tungsten, polishing, anisotropy, contact fatigue, sub surface deformation, tritium retention.

\section{Introduction}

Polishing of tungsten is important for its use as substrate in electronic applications as well as for its use as the plasma facing material in future fusion reactors [1-5]. A considerable number of studies, mainly for the field of electronics, has been devoted to the effect of the polishing parameters. Very flat surfaces can be obtained by the so called chemical-mechanical polishing methods in which an oxidizing chemical reagent is added to a diamond suspension [5-8]. Recently it has been demonstrated that chemical-mechanical polishing leads to a considerable reduction of the surface layer affected by plastic deformation [6]. Much less polishing

*Corresponding author: Willy Vandermeulen, visiting scientist, research fields: metals and ceramics properties. studies aiming at the needs of the fusion field are available. The selection of tungsten for this application is based on its high melting point and high atomic mass. However, during exposure to the fusion plasma, deuterium (D) and tritium (T) atoms are implanted in a surface layer of only a few $10 \mathrm{~nm}$ but even at relatively low temperatures these atoms diffuse to depths of several $\mu \mathrm{m}$ leading to a prohibitive storage of radioactive $\mathrm{T}$ atoms. This so called T-retention may cause serious safety problems. Furthermore, both $\mathrm{D}$ and $\mathrm{T}$ lead to surface deterioration by blister formation (Fig. 1). The blisters consist of sub-surface disk-shaped cavities, filled with molecular $\mathrm{D}$ and $\mathrm{T}$ gas under high pressure. Due to this pressure the cavity wall is pushed outward causing surface damage [4]. 


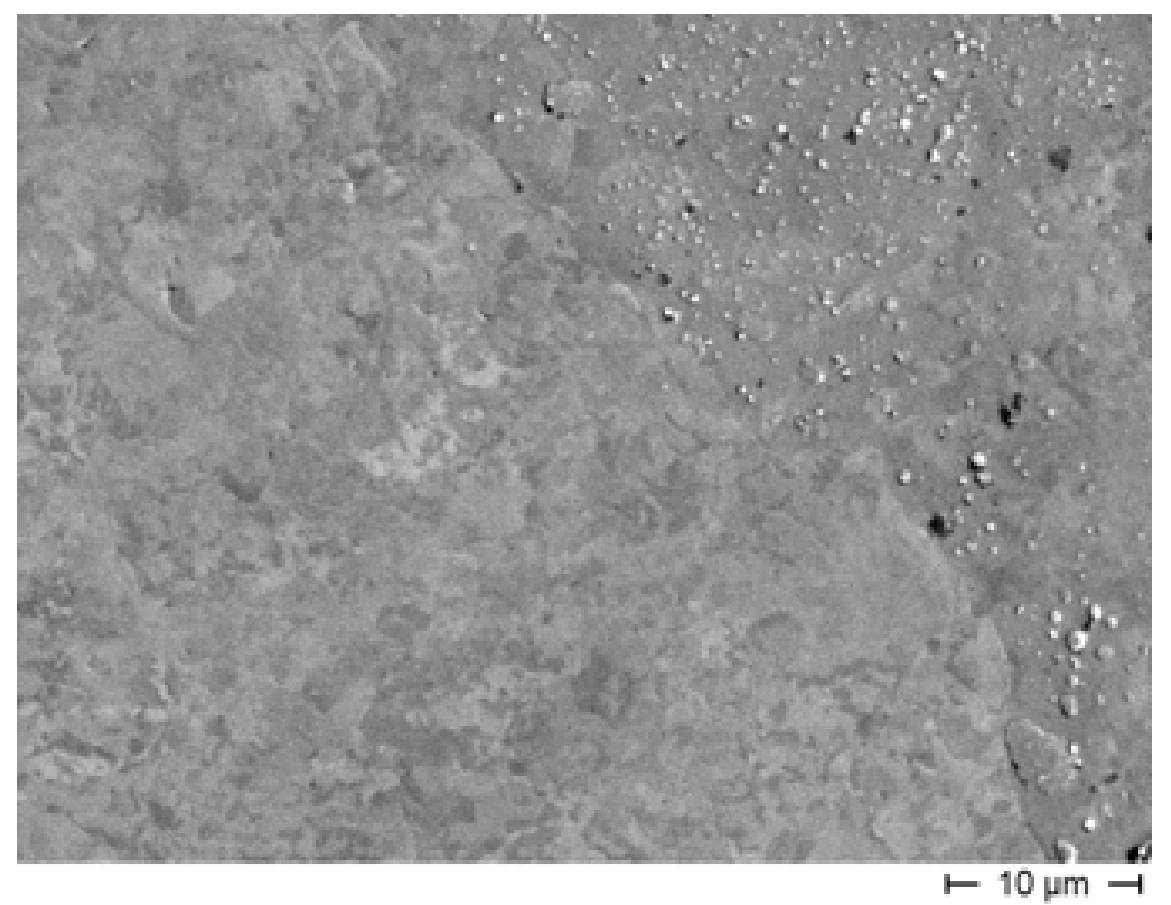

Fig. 1 Mechanically polished tungsten after exposure to a hydrogen plasma. Blister formation in upper right grain. No blisters in left grain.

It is clear that $\mathrm{D} / \mathrm{T}$ retention and blister formation in tungsten are important issues. Therefore, they are extensively studied mostly by exposure to a hydrogen or deuterium plasma. Since the surface layer involved is only of the order of micrometre, the surface preparation method of the specimens used for implantation tests plays an important role. Usually mechanical polishing is used until a mirror aspect is obtained, but of course this does not guarantee that the structure of the near-surface layer is unaffected.

Unfortunately, the laboratories which study the application of tungsten in fusion reactors do not always have the possibility of chemical- mechanical polishing. Moreover, surface oxidation by chemical polishing could also have an unwanted effect on the surface condition. Therefore, for fusion research, tungsten is often polished with conventional metallographic equipment [1, 2].

The short study presented in this article was performed mainly with respect to the field of fusion research in order to draw attention to phenomena which either occur at intermediate polishing steps, but are no more visible after the final step, or which become only apparent after etching. These observations strongly point at the existence of a sub-surface dislocation structure and a surface passivating layer, both resulting from the specimen preparation.

\section{Experimental}

The tungsten used in this study was provided by Plansee in two conditions: recrystallized and forged, with HV20 hardness of 500 and 600 . The grain size of the recrystallized material was of the order of $250 \mu \mathrm{m}$.

Small samples of 3 by $1.5 \mathrm{~mm}$ were mounted separately in Polyfast blocks with a diameter of 25 mm. For grinding, SiC paper 1200 was used. Polishing was done with diamond suspensions of 3 and $1 \mu \mathrm{m}$. The polishing cloth was Struers NAP but DUR was used with the same results. Grinding and polishing was done with Struers TegraPol-11 equipment at a speed of $150 \mathrm{rpm}$ and a force of $15 \mathrm{~N}$. Grinding was done under water flow. For polishing the cloth was kept moist with water. 
The basic polishing sequence consisted of:

- Grinding while rotating the specimen, on 1200 grit paper followed by short (10 sec) manual grinding without rotation to obtain parallel lines. These uniform lines make it easier to follow the subsequent diamond polishing. After grinding, a Vickers hardness indentation was made as a location marker to follow the evolution of the surface appearance.

- Polishing while rotating the specimen, with $3 \mu \mathrm{m}$ grit polycrystalline diamond for times up to $20 \mathrm{~min}$ while rotating the specimen, with $1 \mu \mathrm{m}$ grit polycrystalline diamond for times up to $15 \mathrm{~min}$ optional: etching during $20 \mathrm{~s}$ with 4 parts $\mathrm{H}_{2} \mathrm{O}-25 \% \mathrm{NH}_{3}$ and 1 part $\mathrm{H}_{2} \mathrm{O}_{2}$ to reveal the tungsten grains.

A limited amount of polishing was done with a load of $50 \mathrm{~N}$, on a specimen of 15 by $15 \mathrm{~mm}$ in order to obtain a lower load/unit surface ratio.

The evolution of the surface condition was examined with a Leica DMLM microscope. Since the contrast on normally focused images was very weak it had to be enhanced by under- or over-focusing. These pictures were then further treated with Zeiss-Axiocam-MRc5 software.

\section{Results}

Using the basic preparation procedure described above it was found by optical microscopy (OM) that a satisfactory surface finish could be obtained for forged as well as for recrystallized tungsten. Fig. 2a shows an example of such a surface. It is smooth along the horizontal center zone while at the upper and lower edges some non-smooth regions are probably due to edge effect. In the following, attention will only be paid to the central zone.

Two phenomena will be described. The first consists of surface relief caused by the material removal rate being different from grain to grain. The second is the occurrence of surface cracking observed during the intermediate polishing step with $3 \mu \mathrm{m}$ diamond grit. Both are illustrated and discussed for the recrystallized specimen. The behavior for the forged condition was found to be the same.

To avoid as much as possible pre-existing damage, the preparation was repeated after a preceding polishing sequence up to $1 \mu \mathrm{m}$ (Fig. 2a). This surface was then ground without rotation on paper 1200 and a HV20 indentation was made for reference (Fig. 2b). Due to this grinding a previous indention (marked A, visible on the left side of Fig. 2c), decreased in size due to removal of surface material. The size decrease corresponds to the removal of about $30 \mu \mathrm{m}$.

The ground surface was then polished for $20 \mathrm{~min}$ with $3 \mu \mathrm{m}$ grit diamond (Figs. 2c and 2d). It can be seen that the grains have become visible because each grain shows a particular surface texture. Fig. 3 shows a detail of such a texture. A few regions are smooth but most of the surface shows a dot pattern. In the regions indicated by arrows, the dots can be seen to be aligned in rows. The dot density and the direction of the rows is clearly determined by the orientation of the grain in which they occur.

The observations described above were obtained with a polishing load of $15 \mathrm{~N}$ and specimens of $1.5 \times$ $3 \mathrm{~mm}^{2}$. It was found that after polishing samples of 15 $\times 15 \mathrm{~mm}^{2}$ with the same load, no dot formation occurred. Increasing the load on these samples to $50 \mathrm{~N}$ (maximum available) did restart dot formation.

After further polishing the small specimens with 1 $\mu \mathrm{m}$ grit the dots have disappeared and the surface is featureless on focused optical images (Fig. 2e). However, de-focusing shows that the same relief as found after $3 \mu \mathrm{m}$ polishing is still present (Fig. 2f).

After etching the polished surface, grain boundaries are clearly visible (Figs. $2 \mathrm{~g}$ and $2 \mathrm{~h}$ ). Some grains show an etching pattern and scratch lines while the surface of others remains featureless (Fig. 2h). Comparison of Figs. $2 \mathrm{f}$ and $2 \mathrm{~g}$ confirms that different dot patterns and surface relief corresponds with different grains.

After etching, the sample was re-polished for $5 \mathrm{~min}$ with $1 \mu \mathrm{m}$ grit. This was sufficient to obtain again a 

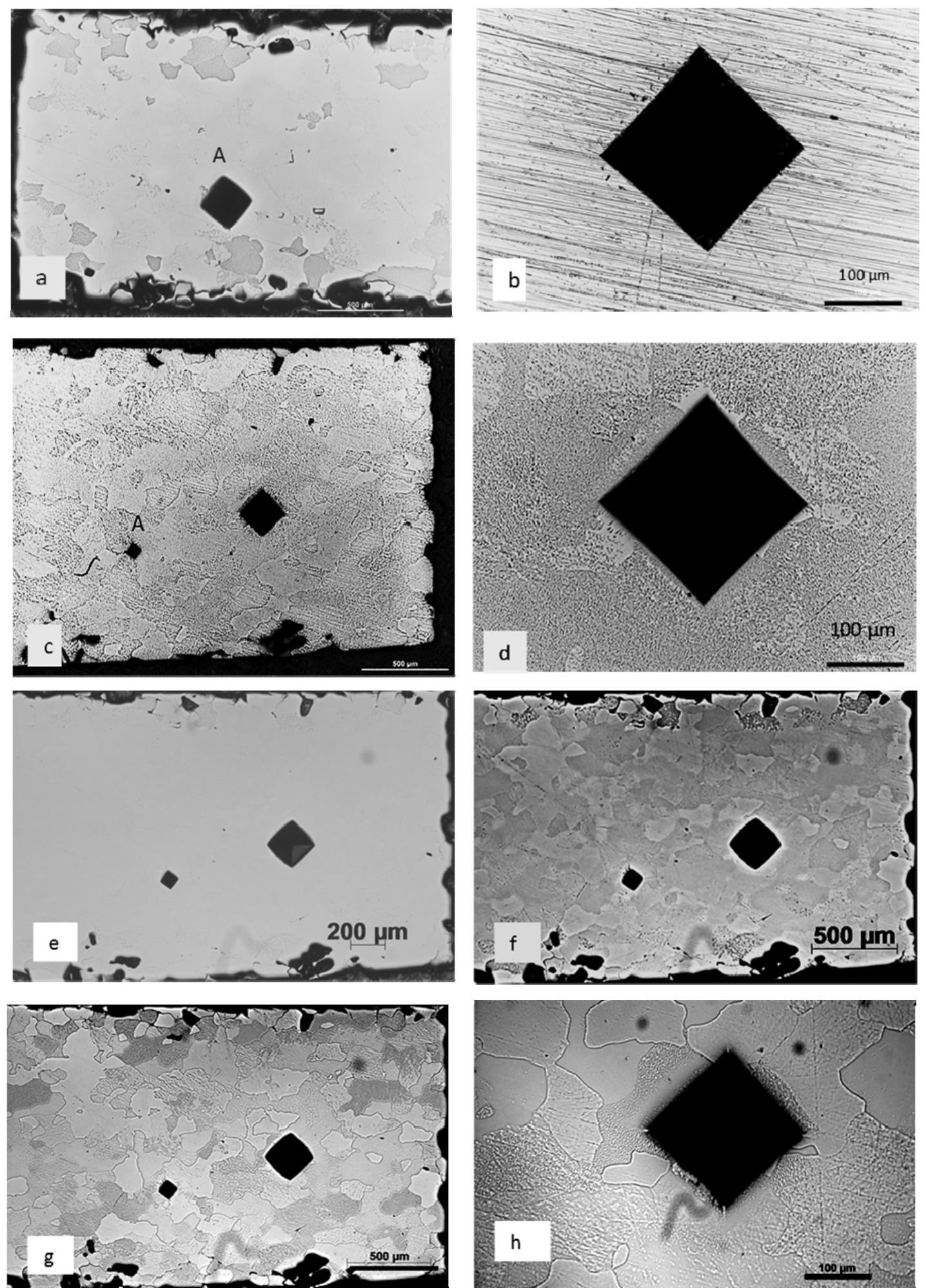

Fig. 2 Evolution of surface upon grinding, polishing and etching: (a) starting condition, (b) manually ground without rotation, (c) polished 20 min with 3 m diamond (d) same, detail of the HV. Note extensive dot formation (e) polished with 1 m diamond, image focused (f) same, over-focused (g) etched. Note boundary and surface attack of most grains and (h) same, detail of $\mathrm{HV}$. 


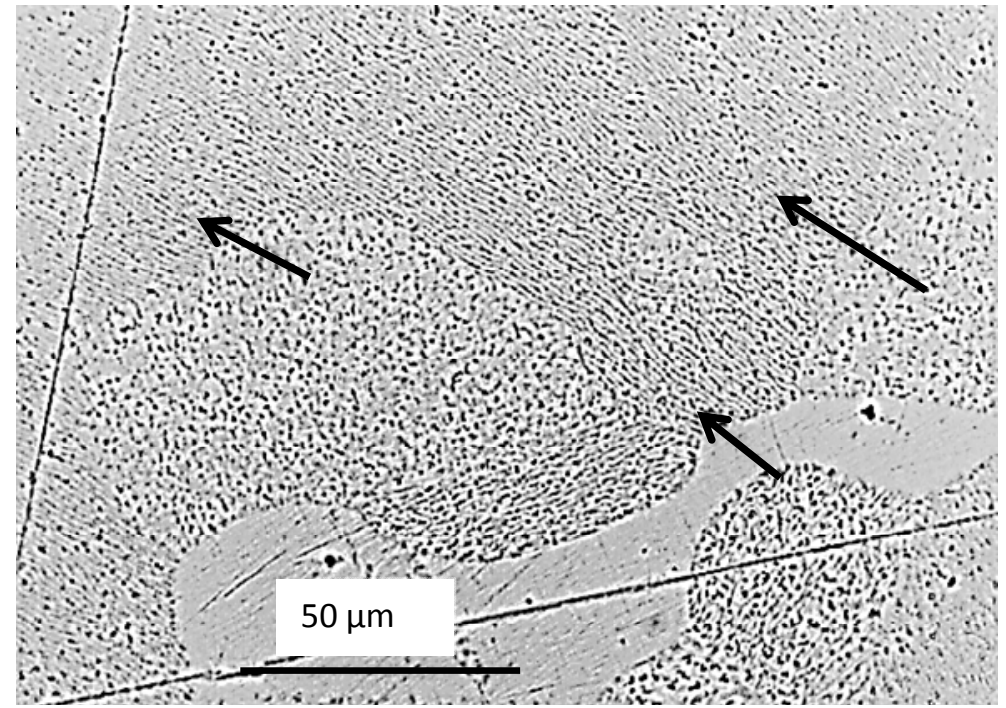

Fig. 3 Surface aspect after $3 \mu \mathrm{m}$ diamond polishing. Note well aligned dots (arrows) and smooth region in lower half.

featureless surface. Subsequently it was polished with the $3 \mu \mathrm{m}$ grit for $5 \mathrm{~min}$. This made the dot patterns appear again.

The dot formation was examined in some detail with a scanning electron microscope (SEM). Fig. 4a shows a surface which was polished initially with 1 $\mu \mathrm{m}$ grit and then kept manually for only $30 \mathrm{~s}$ with a high force on the $3 \mu \mathrm{m}$ polishing disk. No rotation was done in order to mark the polishing direction. It can be seen that cracks with a length of 1-2 $\mu \mathrm{m}$ have appeared. This is more clearly visible on Fig. 4b where the cracks are better visible because they show a bright edge contrast. It should be noted that the traces caused by the scraping diamonds are less than $0.1 \mu \mathrm{m}$ wide although they are caused by $3 \mu \mathrm{m}$ particles. This indicates that the cracks form by lengthwise growth and do not form by single incidents such as by rolling of a large particle over the surface. The presence of the scraping traces also indicates that the polishing mechanism involves plastic deformation and is not due to a brittle mechanism [9].

Fig. 4c shows the surface condition after 20 min polishing with $3 \mu \mathrm{m}$ grit (to be compared with $\mathrm{OM}$ pictures as e.g. Fig. 3). The cracks have now opened to around $0.5 \mu \mathrm{m}$. Finally, Fig. 4d shows the difference in crack formation in two different grains. From this picture it is obvious that the crack orientations are grain dependent.

Electro polishing of tungsten may give rise to the formation of crystallographic facets which in principle allow determining the grain orientation. It was tried if the etchant used in this study also might cause such facets. Therefore, a sample, polished to $1 \mu \mathrm{m}$, was attacked for 30 min. Figs. 5a and 5b show SEM pictures of the etched sample. Most grains are uniformly attacked but the material removal differs from grain to grain. At higher magnification it could be seen that the pattern on the attacked surface is different in different grains but no crystallographic planes were found. The small scale roughness seems to be caused by gas development. On the left side of Fig. 5a, it can be seen that some grains are only locally attacked. Part of their surface has remained almost unaffected; other parts consist of round, deeply etched zones.

Figs. 5a and 5b also show that after this deep etching, indications of grinding traces are still prominent in the sub-surface region of most grains notwithstanding the polished surface was smooth and featureless. The thickness of the material layer removed by etching depends on the grain orientation but is estimated to be about $4 \mu \mathrm{m}$. This shows that even under a smoothly polished surface considerable plastic deformation due to cutting or grinding is still present. 

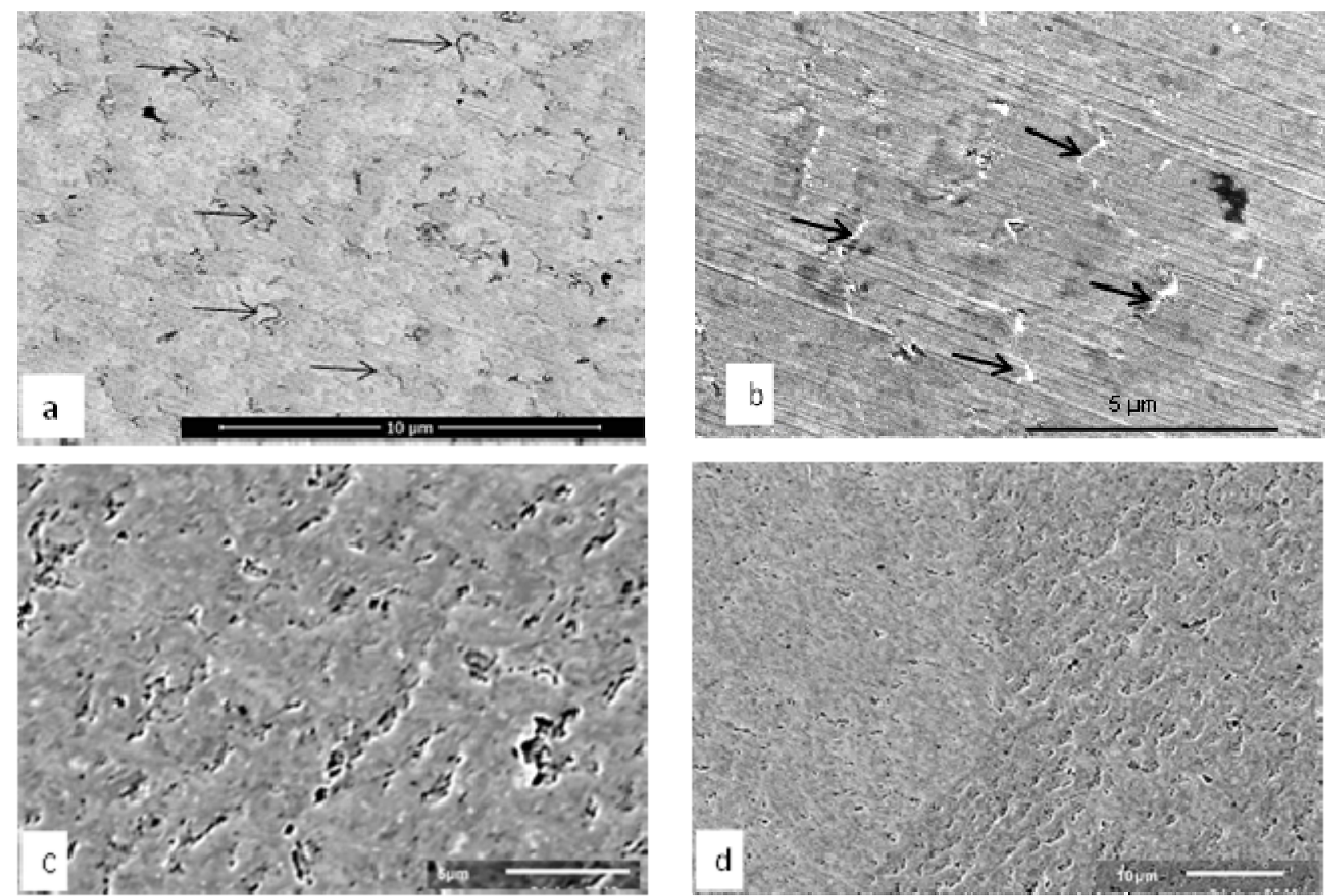

Fig. 4 Evolution of $1 \mu \mathrm{m}$ polished surface, exposed to $3 \mu \mathrm{m}$ diamonds (a) $30 \mathrm{~s}$ with high force, no rotation. Note small cracks indicated by arrows, (b) higher magnification. Arrows indicate cracks. Note parallel polishing traces less than $0.1 \mu \mathrm{m}$ wide, (c) same after 20 min machine polishing with rotation. Note opening of cracks (d) cracks in two different grains, showing grain dependence of the cracking pattern.
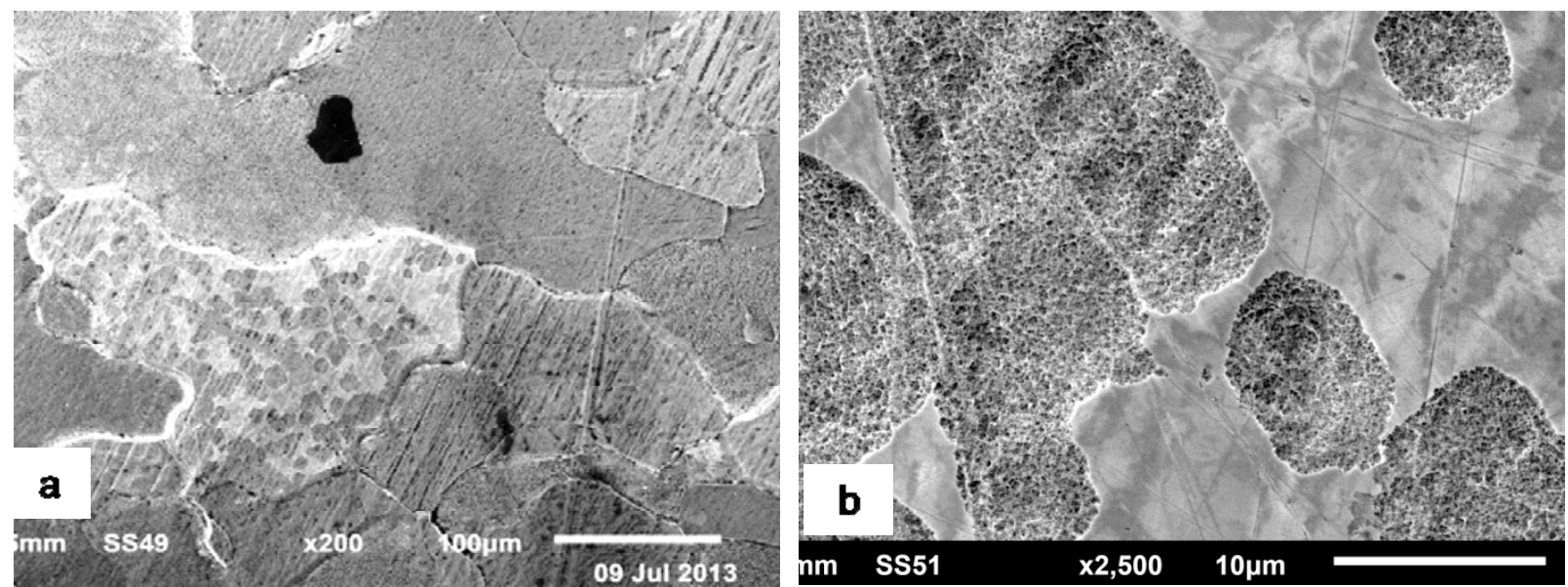

Fig. 5 Polished surface after etching for $30 \mathrm{~min}$ : (a) grain structure revealed because different grains have been attacked to different depths. Grain in the left lower quadrant is only locally attacked. Note grinding traces revealed by etching in most of the grains, (b) detail showing circular attacked holes and grinding traces. 


\section{Discussion}

The above results show that after final polishing to $1 \mu \mathrm{m}$ the surface is not completely planar. Indeed, it can be seen that some grains protrude more than others. Such relief is well known for samples consisting of phases with different hardness. In single phase materials it is not common. In the present case (single phase tungsten) the phenomenon has to be ascribed to the grain orientation dependence of the mechanical properties. Tensile tests on single crystals in the $<110>$ and $<111>$ directions showed an elongation at rupture of the order of $20 \%$ and a fully ductile fracture mode. The elongation in the $<100>$ direction was only about $5 \%$ and cleavage fracture occurred. In addition, the proportional limit in the $<100>$ orientation is only about one third of the $<110>$ and <111> directions [10]. Since the polishing mechanism consists of scraping of the metal with diamond particles as can be seen from Fig. 4b, material removal is controlled by the ease of plastic deformation. Although deformation by scraping is much more complex than in a tensile test it can be understood that anisotropic tensile properties entail anisotropic resistance to material removal by polishing. A second factor which may cause relief is the formation of an oxide layer. Such a layer was observed under conditions of chemical-mechanical polishing and the brittleness of a chemically passivating layer has been suggested to contribute to the polishing mechanism [8]. It can be assumed that an oxide layer also forms during wet polishing. If the characteristics of such a layer are orientation dependent this may also contribute to grain dependent relief.

The observed relief is not expected to have a direct effect on the $\mathrm{D} / \mathrm{T}$ implantation process. Much more important is the material condition at and below the surface. Information about this condition can be deduced from the deeply etched sample shown by Fig. 5. Traces of grinding lines can still be seen in many grains. This shows that after mechanical surface preparation, plastic deformation persists to depths of the same order as the diffusion range of implanted ions, even below smoothly polished surfaces. It can be expected that even after chemical-mechanical polishing or ion cutting plastic deformation from previous surface machining will be present if insufficient material has been removed.

Figure 5 gives also information about the condition at the surface. Although the etching was quite long, some grains show only circular, deeply attacked zones. This suggests that those grains were protected by a surface layer containing weak spots. On the other hand, since most of the specimen was attacked it is evident that the protective quality of such a layer is limited to specific grain orientations.

The second phenomenon considered in this polishing study is the formation of cracks by $3 \mu \mathrm{m}$ grit diamond. This cracking is favored by a high polishing force and a small sample size. It strongly depends on grain orientation. This phenomenon can be explained by a mechanism similar to sliding contact fatigue [11]. In the present case the sliding action is performed by the diamond particles which cause very high alternating contact stresses at each point of the surface. The scraping action of the diamonds provides the necessary shearing force for the surface pitting and cracking as described in [11]. Similar to the grain orientation related relief it can be understood that the amount of cracking and the arrangement of the cracks are dominated by the mechanical property anisotropy. The fact that no cracking occurs with $1 \mu \mathrm{m}$ grit can be understood by supposing that the contact forces exerted by this grit size are too small to cause fatigue damage. The suppression of crack formation by increasing the specimen surface at a fixed load can be understood in the same way.

With respect to implantation tests, Fig. 1 shows that blister formation also depends on the grain orientation. This could be a purely grain orientation controlled effect but it is not excluded that blistering is 
also affected by the surface and sub surface defect state. Therefore, careful characterization of this state has to be strongly recommended for $\mathrm{H} / \mathrm{D} / \mathrm{T}$ implantation and retention studies.

\section{Conclusions}

The surface relief found on mechanically polished tungsten is explained by the anisotropy of the mechanical properties. The formation of an orientation dependent oxide layer might also contribute to the relief formation.

The grain dependent pattern of surface cracks, caused by polishing with $3 \mu \mathrm{m}$ grit diamond, is caused by sliding contact fatigue under a high specific loading.

Both, the occurrence of polishing marks on deeply etched surfaces and the observation of fatigue deformation, are indications that plastic deformation due to the sample preparation is present below apparently good polished surfaces. This shows the necessity of a careful characterization of the surface and sub-surface defect structure of specimens used for $\mathrm{H} / \mathrm{D} / \mathrm{T}$ retention studies.

\section{References}

[1] Zayachuk, Y., 't Hoen, M. H. J., Zeijlmans van Emmighoven, P. A., Terentyev, D., Uytdenhouwen, I. and van Oost, G. 2013. "Surface Modification of Tungsten and Tungsten-Tantalum Alloys Exposed to High-Flux Deuterium Plasma and its Impact on Deuterium Retention.” Nucl. Fusion 53: 0130131-7.

[2] Alimov, V. Kh., Tyburska-Puschel, B., Lindig, S.,
Hatano, Y., Balden, M. and Rith, J. et al. 2012. "Temperature Dependence of Surface Morphology and Deuterium Retention in Polycrystalline ITER-Grade Tungsten Exposed to Low-Energy, High-Flux D Plasma.” J. Nucl. Mater. 420: 519-24.

[3] Manhard, A., Matern, G. and Balden, M. 2013. “A Step-by-Step Analysis of the Polishing Process for Tungsten Specimens” Pract. Metallogr. 50: 5-16.

[4] Jia, Y. Z., De Temmerman, G., Luo, G.-N., Xu, H. Y., Li, C. and Fu, B. Q. et al. 2015. "Surface Morphology and Deuterium Retention in Tungsten Exposed to High Flux D Plasma at High Temperatures” J. Nucl. Mater. 457: 213-9.

[5] Stojadinovic, J. 2009. "Chemical-Mechanical Polishing of Tungsten: Electrochemical and Tribo-Corrosion Approach.” Ph.D. thesis, Federal Polytechnical School of Lausanne, Switserland.

[6] Bielmann, M., Mahajan, U. and Singh, R. K. 1999. "Effect of Particle Size during Tungsten Chemical Mechanical Polishing.” Electrochemical and Solid-State Letters 2: 401-3.

[7] Larsen-Basse, J. and Liang, H. 1999. "Probable Role of Abrasion in Chemo-Mechanical Polishing of Tungsten.” Wear 233-235: 647-54.

[8] Lim, M. S., van der Heide, P. A. W., Perry, S. S., Galloway, H. C. and Koeck, C. K. 2004. "Microscopic Investigations of Chemo-Mechanical Polishing of Tungsten.” Thin Solid Films 457: 346-53.

[9] Samuels, L. E. 1982. "Metallographic Polishing by Mechanical Methods.” In American Society for Metals. Chap.7: Brittle Materials, Metals Park, Ohio.

[10] Beardmore, P. and Hull, D. 1965. "Deformation and Fracture of Tungsten Single Crystals”. J. Less-Common Metals 9: 168-80.

[11] Sadeghi, F., Jalalahmadi, B., Slack, T. S., Raje, N. and Arakere, N. K. 2009. "A Review of Rolling Contact Fatigue.” J. of Tribology 131: 0414031-15. 